\title{
Further study of a reversing visual illusion
}

\author{
W. H. TEDFORD, JR., and MARYNELL MURPHY \\ Southern Methodist University, Dallas, Texas 75275
}

\begin{abstract}
Subjects compared an open interval, bounded by vertical lines, to one of equal length which contained an intervening stimulus. The intervening stimulus increased perceived length for small visual angles but decreased it for larger angles. Reversal occurred at approximately the point at which both end markers could no longer be encompassed in one fixation. Increasing the size of the intervening stimulus increased the magnitude of the illusion in both directions.
\end{abstract}

Tedford and Gray (1976) have shown that when the open space between two vertical lines is interrupted by a dot, the perceived distance between the lines is altered. When the actual distance is short, the intervening stimulus increases the perceived distance; however, when the distance is longer, the illusion reverses and the intervening stimulus decreases the perceived distance.

The present study extends these results by attempting to locate the reversal point. A possible interpretation in terms of visual span is offered.

\section{METHOD}

\section{Subjects}

Volunteers from undergraduate psychology classes were tested individually; 100 subjects (68 females) were used in the first experiment and 25 (14 females) in the second.

\section{Apparatus}

Stimuli were 50 pairs of $12.7 \times 20.3 \mathrm{~cm}$ cards. Centered on each card was a pair of $3-\mathrm{cm}$ vertical lines, separated by varying distances. One card in each pair had a square or rectangle between the vertical lines. For 16 pairs, separation distance varied from 1 to $16 \mathrm{~cm}$, with the intervening stimulus being a square $1 \mathrm{~cm}$ on each side. A second series varied distance from 2 to $16 \mathrm{~cm}$ and used a rectangle $1 \times 2 \mathrm{~cm}$. A third series varied distance from 3 to $16 \mathrm{~cm}$, with a $1 \times 3 \mathrm{~cm}$ rectangle. Finally, there were five pairs in which the empty interval was not equal to the filled interval: $1.5 \mathrm{~cm}$ vs. $1 \mathrm{~cm}$ (with $1-\mathrm{cm}$ square), $7 \mathrm{vs}$. 8 (with square), 9 vs. 8 (with square), 2.5 vs. 3 (with $1 \times 2$ rectangle), 14 vs. 15 (with $1 \times 3$ rectangle).

Pairs of cards were viewed monocularly at $100 \mathrm{~cm}$ (Experiment 1) or $75 \mathrm{~cm}$ (Experiment 2) through a circular opening equipped with a shutter.

\section{Procedure}

Assignment of the empty distance in each pair to the right or left side was random; assignment was reversed with alternate subjects. Subjects were given a warning signal and then a 1-sec exposure to each pair of cards. They recorded their own judgment of which distance was longer by circling $L$ or $R$ on a record sheet.

\section{RESULTS}

The percentage of subjects selecting each filled interval was calculated and a least-squares fit to the data was made for each of the three main series. The curves

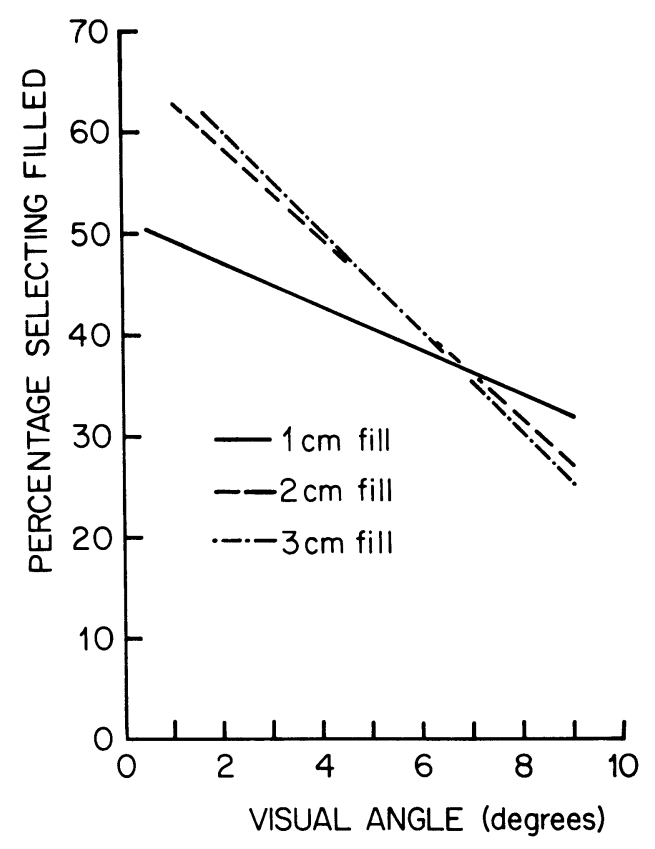

Figure 1. Percentage of subjects who judged a distance with an intervening stimulus to be longer than an empty distance of equal length.

for the first experiment are shown in Figure 1, and the intercepts and slopes for both experiments are given in Table 1 .

A rough estimate of the magnitude of the illusion can be made from the five unequal pairs. Only in the 14 (empty) vs. 15 (filled) pair was an actual difference in length overcome by the illusion, with $56 \%$ of the subjects selecting the $14-\mathrm{cm}$ distance as longer.

Table 1

Straight-Line Equations for Both Experiments

\begin{tabular}{cccccc}
\hline \multirow{2}{*}{$\begin{array}{c}\text { Fill } \\
(\mathrm{cm})\end{array}$} & \multicolumn{2}{c}{$y$-Intercept $(\%)$} & & \multicolumn{2}{c}{ Slope $(\% / \mathrm{deg})$} \\
\cline { 2 - 3 } \cline { 6 - 6 } & First & Second & & First & Second \\
\hline 1 & 51.2 & 49.3 & & 2.2 & 1.0 \\
2 & 67.7 & 63.0 & & 4.5 & 3.0 \\
3 & 70.0 & 75.8 & & 4.9 & 4.5 \\
\hline
\end{tabular}


Percentages for the other empty intervals were: 1.5 (89\%), $2.5(17 \%), 7(24 \%)$, and $9(83 \%)$. This indication that the illusion had a small magnitude was supported by data from five subjects who were not required to make a forced choice; approximately $80 \%$ of their judgments were "equal." Data were examined for sex differences and side biases. None were found.

\section{DISCUSSION}

In both experiments, the cross-over point for the two series with rectangular fills is 4 to 5 deg of visual angle. For the square fill, both experiments give a value of less than $1 \mathrm{deg}$. However, visual inspection of the data indicates that they conform less well to a straight line; they seem to drop off more sharply at the short end. Finding a "best fit" with a French curve gives a crossover of around $3 \mathrm{deg}$. The previous study (Tedford \& Gray, 1976) estimated $9 \mathrm{deg}$ as the cross-over point, but it used a circular fill and presented the pairs one above the other, with the endpoints offset.

Both studies found a great deal of between-subjects variation. However, when the record for a single subject is examined, the consistency of responding is quite high. If a cross-over point is selected for an individual record, about $90 \%$ of the responses are consistent with it.

The authors believe that the reversal can best be accounted for in terms of the subject's visual span. If both end markers are seen in the same fixation, then the intervening stimulus tends to break up the pattern and increase the perceived distance. If the distance is so great that the subject fixates on one end marker and then sweeps to a fixation on the other, encountering the stimulus seems to make the perceived sweep shorter. A value of 3 to 9 deg for visual span is in line with the results of Weber (1942). This also explains the variation in responding, since each subject responds in terms of an individual visual span.

It seems fairly safe to conclude that increasing the size of the intervening stimulus increases the magnitude of the illusion in both directions, thus increasing the slope of the curve. This is probably true only as long as the height of the end markers remains constant.

\section{REFERENCES}

Tedford, W. H., JR., \& GRAY, C. F. Reversal of a visual illusion of length perception. Bulletin of the Psychonomic Society, 1976, 7, 63-64.

WEBER, C. O. Effects of practice on the perceptual span for letters. Journal of General Psychology, 1942, 26, 347-351.

(Received for publication May 22, 1978.) 